WISC-MILW-95-TH-17

\title{
Hawking Radiation and Unitary Evolution
}

\author{
Sukanta Bose, ${ }^{*}$ Leonard Parker, ${ }^{\dagger}$ and Yoav Peleg ${ }^{\ddagger}$ \\ Department of Physics \\ University of Wisconsin-Milwaukee, P.O.Box 413 \\ Milwaukee, Wisconsin 53201, USA
}

\begin{abstract}
We find a family of exact solutions to the semi-classical equations (including back-reaction) of two-dimensional dilaton gravity, describing infalling null matter that becomes outgoing and returns to infinity without forming a black hole. When a black hole almost forms, the radiation reaching infinity in advance of the original outgoing null matter has the properties of Hawking radiation. The radiation reaching infinity after the null matter consists of a brief burst of negative energy that preserves unitarity and transfers information faster than the theoretical bound for positive energy.
\end{abstract}

\footnotetext{
*Electronic address: bose@csd.uwm.edu

†Electronic address: leonard@cosmos.phys.uwm.edu

‡Electronic address: yoav@csd.uwm.edu
} 
Recently we presented a modified two-dimensional (2D) dilaton gravity theory that is exactly solvable semiclassically [1]. In this 2D theory (as in 4D Einstein gravity), infalling null matter forms a black hole only if its energy $M$ is above a certain critical value $M_{c r}$. In Ref. [1] we studied the supercritical case, $M>M_{c r}$, in which a black hole is formed and evaporates by emitting Hawking radiation. In this work we study the subcritical case, $M<M_{c r}$, in which the infalling matter becomes outgoing and escapes to infinity without forming a black hole. Because we can obtain the numerical solution to the future of the classical outgoing matter, we can see how correlations among outgoing created particles preserve unitarity and how Hawking radiation originates as the energy of the infalling matter approaches $M_{c r}$.

The effective action of the modified 2D dilaton gravity theory is [1]

$$
\begin{aligned}
S & =\frac{1}{2 \pi} \int d^{2} x \sqrt{-g(x)}\left[\left(e^{-2 \phi}-\kappa \phi\right) R(x)\right. \\
& \left.+\left(4 e^{-2 \phi}+\kappa\right)(\nabla \phi)^{2}+4 \lambda^{2} e^{-2 \phi}-\frac{1}{2} \sum_{i=1}^{N}\left(\nabla f_{i}\right)^{2}\right] \\
& -\frac{\kappa}{8 \pi} \int d^{2} x \sqrt{-g(x)} \int d^{2} x^{\prime} \sqrt{-g\left(x^{\prime}\right)} R(x) G\left(x, x^{\prime}\right) R\left(x^{\prime}\right),
\end{aligned}
$$

where $\phi$ is the dilaton field, $R(x)$ is the $2 \mathrm{D}$ Ricci scalar, $\lambda$ is a constant, $f_{i}$ are $N$ matter (massless scalar) fields, $\kappa=\hbar N / 12$, and $G\left(x, x^{\prime}\right)$ is an appropriate Green function for $\nabla^{2}$ (for more details see Ref. [1]). In null coordinates, $z^{ \pm}$, and a conformal gauge, $g_{++}=g_{--}=0$, $g_{+-}=\frac{1}{2} e^{2 \rho}$, the equations of motion (from varying $f_{i}, \phi$ and $g_{+-}$) take the form $\partial_{+} \partial_{-} Y=$ $0=\partial_{+} \partial_{-} f_{i}$ and $\partial_{+} \partial_{-} X=-\lambda^{2} e^{-2 Y}$, where $X \equiv e^{-2 \phi}$ and $Y \equiv \phi-\rho$. The constraints (from varying $g_{++}$and $\left.g_{--}\right)$are $-\partial_{ \pm}^{2} X-2 \partial_{ \pm} X \partial_{ \pm} Y-\left(T_{ \pm \pm}^{f}\right)_{c \ell}+\kappa\left[\left(\partial_{ \pm} Y\right)^{2}+\partial_{ \pm}^{2} Y+t_{ \pm}\left(z^{ \pm}\right)\right]=0$, where $\left(T_{ \pm \pm}^{f}\right)_{c \ell}=\frac{1}{2} \sum_{i=1}^{N}\left(\partial_{ \pm} f_{i}\right)^{2}$ is the classical (zero order in $\hbar$ ) contribution to the energymomentum tensor of the $f_{i}$ matter fields, and $t_{ \pm}\left(z^{ \pm}\right)$are integration functions determined by the specific quantum state of the matter scalar fields [回]. Because $\phi$ is a scalar, but $\rho$ is a function of $g_{+-}$and $\partial_{+} \partial_{-} Y=0$, it is possible to define coordinates $x^{+}\left(z^{+}\right)$and $x^{-}\left(z^{-}\right)$in which $Y \equiv \phi-\rho$ is 0 . In this work we use these "Kruskal" coordinates $x^{+}, x^{-}$.

A general solution in the Kruskal gauge for the $X$-field equation of motion and constraints 
is

$$
\begin{aligned}
X\left(x^{+}, x^{-}\right)= & -\lambda^{2} x^{+} x^{-}-\int^{x^{+}} d x_{2}^{+} \int^{x_{2}^{+}} d x_{1}^{+}\left[\left(T_{++}^{f}\right)_{c \ell}-\kappa t_{+}\left(x_{1}^{+}\right)\right] \\
& -\int^{x^{-}} d x_{2}^{-} \int^{x_{2}^{-}} d x_{1}^{-}\left[\left(T_{--}^{f}\right)_{c \ell}-\kappa t_{-}\left(x_{1}^{-}\right)\right] .
\end{aligned}
$$

The vacuum solutions are the ones for which $\left(T_{ \pm \pm}^{f}\right)_{c \ell}=0$ and $t_{ \pm}\left(x^{ \pm}\right)=\left(2 x^{ \pm}\right)^{-2}$ [1]. They are static solutions because in the asymptotically flat coordinates $\sigma^{ \pm}=t \pm \sigma= \pm \lambda^{-1} \log \left( \pm \lambda x^{ \pm}\right)$, they take the $t$-independent form $X_{v a c}(\sigma)=e^{2 \lambda \sigma}-\kappa \lambda \sigma / 2+C$, where $C$ is a constant. For $C>C^{*} \equiv(\kappa / 4)(\log (\kappa / 4)-1)$ the vacuum solutions have a null singularity at $x^{ \pm}=0$, which is a finite affine distance from any point in the interior of the space-time. For $C<C^{*}$ the vacuum solutions have a time-like (naked) singularity on the curve $\sigma=\sigma_{s}$, for which $X_{v a c}\left(\sigma_{s}\right)=0$. The solution with $C=C^{*}$ is a semi-infinite throat which is everywhere regular and geodesically complete [1].

In addition to initial conditions giving $\left(T_{++}^{f}\right)_{c \ell}$ and $t_{+}\left(x^{+}\right)$on asymptotic past null infinity, $\Im^{-}$, we must also determine $\left(T_{--}^{f}\right)_{c \ell}$ and $t_{-}\left(x^{-}\right)$. To do so, we choose reflecting boundary conditions on the matter fields $f_{i}$ [2 25]. We consider very localized infalling matter for which $\left(T_{++}^{f}\right)_{c \ell}\left(x^{+}\right)$is non-zero only for $\left|x^{+}-x_{0}^{+}\right|<\epsilon$, with $\lambda \epsilon<<1$ (see Fig. 四). We take $t_{+}\left(x^{+}\right)=\left(2 x^{+}\right)^{-2}$ everywhere, which corresponds to no quantum radiation on $\Im^{-}$.

The reflecting boundary conditions are imposed at the boundary curve on which $X\left(x^{+}, x^{-}\right) \equiv X_{B}=$ constant. We take $X_{B} \geq 0$ so that the dilaton field $\phi=-\frac{1}{2} \log (X)$ is real. For a dynamical boundary curve, following a general trajectory $x^{+}=x_{B}^{+}\left(x^{-}\right) \equiv p\left(x^{-}\right)$, the reflecting boundary condition is [2 -5]

$$
T_{--}^{f}=\left(p^{\prime}\right)^{2} T_{++}^{f}+\kappa\left(p^{\prime}\right)^{1 / 2} \partial_{-}^{2}\left(p^{\prime}\right)^{-1 / 2}
$$

where $^{\prime}=\partial / \partial x^{-}$, and $T_{\mu \nu}^{f}$ is the energy-momentum tensor of the matter fields, including both the classical and one-loop contributions. Namely, $T_{\mu \nu}^{f}=\left(T_{\mu \nu}^{f}\right)_{c \ell}+\left\langle T_{\mu \nu}\right\rangle$, where $\left\langle T_{ \pm \pm}\right\rangle=$ $\kappa\left[\partial_{ \pm}^{2} \rho-\left(\partial_{ \pm} \rho\right)^{2}-t_{ \pm}\left(x^{ \pm}\right)\right]$is the one-loop contribution to the energy-momentum tensor of the scalar matter fields [6]. The last term on the right-hand-side of Eq. (3) arises because of quantum particle creation from the boundary (which is effectively a "moving mirror" [2]). 
The classical part of $T_{\mu \nu}^{f}$ obeys $\left(T_{--}^{f}\right)_{c \ell}=\left(p^{\prime}\right)^{2}\left(T_{++}^{f}\right)_{c \ell}$, which is equivalent to the fields $f_{i}$ satisfying either Neumann or Dirichlet boundary condition. While the quantum part obeys $\left\langle T_{--}\right\rangle=\left(p^{\prime}\right)^{2}\left\langle T_{++}\right\rangle+\kappa\left(p^{\prime}\right)^{1 / 2} \partial_{-}^{2}\left(p^{\prime}\right)^{-1 / 2}$. The boundary condition (3) is also conformally invariant [7]:3].

To the past of the infalling null matter, $x^{+}<x_{0}^{+}-\epsilon$, i.e., region I in Fig. 1, the geometry is one of the static vacuum solutions. The reflecting boundary condition (3) implies that $\left(T_{--}^{f}\right)_{c \ell}\left(x^{-}\right)=0$ and $t_{-}\left(x^{-}\right)=\left(2 x^{-}\right)^{-2}$ for $x^{-}<x_{B}^{-}\left(x_{0}^{+}-\epsilon\right)$. The solution (22) to the past of the outgoing classical null matter, regions I and II in Fig. 田, is therefore

$$
X_{<}\left(x^{+}, x^{-}\right)=-x^{+}\left(\lambda^{2} x^{-}+P_{+}\left(x^{+}\right)\right)-\frac{\kappa}{4} \log \left(-\lambda^{2} x^{+} x^{-}\right)+\frac{M\left(x^{+}\right)}{\lambda}+C,
$$

where $M\left(x^{+}\right)=\lambda \int^{x^{+}} x_{1}^{+}\left(T_{++}^{f}\right)_{c \ell}\left(x_{1}^{+}\right) d x_{1}^{+}$and $P_{+}\left(x^{+}\right)=\int^{x^{+}}\left(T_{++}^{f}\right)_{c \ell}\left(x_{1}^{+}\right) d x_{1}^{+}$are the mass and momentum of the classical infalling matter.

For initial static geometries with $C<C^{*}$, the (very localized) infalling matter forms a black hole only if $M>M_{c r} \equiv-\lambda^{3} x_{0}^{+} x_{0}^{-}-\kappa \lambda / 4$, where $M=M\left(x^{+}>x_{0}^{+}+\epsilon\right)$ is the total mass of the infalling matter, and $x_{0}^{-}=x_{B}^{-}\left(x_{0}^{+}\right)$. The black hole evaporates by emitting Hawking radiation [1], and the semiclassical evolution seems to be non-unitary [1];8]. In the subcritical case, $M<M_{c r}$, the classical infalling matter is reflected from the boundary, which is always time-like (see Fig. 1) and the evolution is unitary. We next find the solution numerically to the future of the outgoing classical null matter (i.e., in region III of Fig.四) in this subcritical case.

In Kruskal coordinates we use the constraint equations to write the boundary condition (3) (on the one loop term) in the form

$$
t_{-}\left(x^{-}\right)=\left(p^{\prime}\right)^{2} t_{+}\left(p\left(x^{-}\right)\right)-\frac{\left(p^{\prime}\right)^{1 / 2} \partial_{-}^{2}\left(p^{\prime}\right)^{-1 / 2}}{1+\kappa /\left(2 X_{B}\right)} .
$$

Since we are considering localized infalling matter, the term $\left(p^{\prime}\right)^{1 / 2} \partial_{-}^{2}\left(p^{\prime}\right)^{-1 / 2}$, which is of the order of $M /\left(\lambda \epsilon^{2}\right)$ in the region of classical reflection, is large. However, in this work we consider the case in which $X_{B}$ is small enough [9] that $X_{B} M /\left(\kappa \lambda^{3} \epsilon^{2}\right)<<1$. This is consistent with the condition, $\hbar / X_{B}<<1$, that is necessary for the semiclassical approximation to 
be valid everywhere, since in the large $N$ limit we can take $\hbar$ to zero sufficiently fast while keeping $\kappa=N \hbar / 12$ constant [1,05.3]. In the limit $X_{B} M /\left(\kappa \lambda^{3} \epsilon^{2}\right) \rightarrow 0$, the final term in Eq. (5) is negligible, and Eq. (5) reduces to $t_{-}\left(x^{-}\right)=\left(p^{\prime}\right)^{2} t_{+}\left(p\left(x^{-}\right)\right)=\left(p^{\prime} / p\right)^{2} / 4$.

One can study the solutions for general infalling matter (not necessarily a very localized one). The main features regarding correlations and Hawking radiation with general infalling matter [10 are already present in this case with $\lambda \epsilon<<1$. In this limit the solution to the future of $x_{0}^{-}$(region III in Fig. 1) can be written in the form

$$
X_{>}\left(x^{+}, x^{-}\right)=-x^{+}\left(\lambda^{2} x^{-}+P_{+}\right)-\frac{\kappa}{4} \log \left(\lambda x^{+}\right)+F\left(x^{-}\right),
$$

where $P_{+}=P_{+}\left(x^{+}>x_{0}^{+}+\epsilon\right)$ is the total momentum of the classical infalling matter and the function $F\left(x^{-}\right)$is determined by the boundary conditions. From (6) and the constraints, we see that $\kappa t_{-}\left(x^{-}\right)=\partial_{-}^{2} F\left(x^{-}\right)$. We define the dimensionless coordinate $y \equiv \lambda x^{-}+P_{+} / \lambda$ and the dimensionless function $q(y) \equiv \lambda x_{B}^{+}\left(x^{-}(y)\right.$ ), and using (6) get an ordinary (second order) differential equation for the boundary curve $\lambda x_{B}^{+}=q(y)$

$$
\left[y q(y)+\frac{\kappa}{4}\right] \frac{d^{2} q}{d y^{2}}=q(y)\left[\frac{\kappa}{2} \frac{1}{q^{2}(y)}\left(\frac{d q}{d y}\right)^{2}-2 \frac{d q}{d y}\right] .
$$

Define $y_{0}=\lambda x_{0}^{+}+P_{+} / \lambda$ to be the value of $y$ just after the classical reflection (in the limit $\lambda \epsilon \rightarrow 0)$. Using the continuity of the solution and the classical reflecting boundary condition, we get the initial data for $q(y)$ : $q\left(y_{0}\right)=\lambda x_{0}^{+}$and $(d q / d y)\left(y_{0}\right)=$ $\lambda x_{0}^{+}\left[M /\left(\lambda y_{0}\right)-\lambda x_{0}^{+}-\left(4 \lambda x_{0}^{-}\right)^{-1}\right] /\left(\lambda x_{0}^{+} y_{0}+\kappa / 4\right)$. We choose $\lambda x_{0}^{+}=-\lambda x_{0}^{-}=1$ and $\kappa=10^{-6}$, and solve (7) numerically for different values of the infalling mass. The results that we get are qualitatively the same for any value of $\kappa$ as long as $\kappa \lambda<<M_{c r}$. We use an embedded fifth order Runge-Kutta ODE integration routine [11]. The solutions for $q(y)$ are shown in Fig. 2 .

When the infalling mass, $M$, is much less than $M_{c r}$, as in Fig. 2al, the boundary curve is hardly affected by the infalling matter, but when $M$ approaches $M_{c r}$, as in Fig. 2 $1 \mathrm{~b}$, the timelike boundary curve is strongly affected and approaches a null curve.

To see that the solutions are indeed stable (i.e., that the total amount of energy radiated to $\Im^{+}$is finite) we calculate the created energy radiated to $\Im^{+},\left\langle T_{--}\right\rangle(u)=\left(\kappa \lambda^{2} / 4\right)[1-$ 
$\left.4 y^{2}(u) t_{-}(y(u))\right]$, where $u=-\lambda^{-1} \log (-y)$ and $v=\lambda^{-1} \log \left(\lambda x^{+}\right)$are null coordinate in which the metric is manifestly asymptotically flat (i.e., $\rho \rightarrow 0$ on $\Im^{+}$). The results are shown in Fig. 3 for the same cases considered in Fig. 2.

The negative-energy radiation to the future of the original outgoing null matter (i.e., in the region $u>u_{0}$ ) approaches zero exponentially fast. The magnitude of the total radiated negative energy is bounded by $\left|\int_{u_{0}}^{\infty}\left\langle T_{--}(u)\right\rangle d u\right|<(\kappa \lambda / 4) \log \left(4 M_{c r} / \lambda \kappa\right)$. If $\Delta t$ is the time as measured by an asymptotic observer in which this energy $E$ is radiated to $\Im^{+}$, then we obtain a quantum inequality, $|E| \Delta t \sim \kappa\left(M_{c r}-M\right)^{2}\left(\log \left[M_{c r} /\left(M_{c r}-M\right)\right]\right)^{2} /\left(M M_{c r}\right)<\kappa=N \hbar / 12$, of the type discussed by Ford and Roman [12]. The Heisenberg time-energy uncertainty principle implies that an attempt to measure the energy of this burst in the available time $\Delta t$ will disturb the energy by at least $|E|$.

We have verified numerically that the magnitude of negative energy radiated to the future of the classical reflected matter $\left(u>u_{0}\right)$ is equal to that of the positive quantum radiation reaching $\Im^{+}$before the classical reflected matter $\left(u<u_{0}\right)$. Thus energy is conserved. The total amount of energy on $\Im^{+}$is just that of the original reflected null matter, $E_{\Im^{+}}^{\text {tot }}=$ $\int_{-\infty}^{\infty}\left(T_{--}^{f}\right)(u) d u=\int_{-\infty}^{\infty}\left(T_{--}^{f}\right)_{c \ell} d u=M$, and the solutions are indeed stable.

In Fig. 3a the mass is far below the critical mass and the quantum flux of radiation is much less than that of thermal Hawking radiation, while in Fig. 3b the flux of radiation before the classical reflection (for $u$ in the range $u_{0}-\lambda^{-1}<u<u_{0}$ ) approaches that of Hawking radiation. As $M$ approaches the critical mass, the radiation before the classical reflection becomes indistinguishable from Hawking radiation originating from a black hole.

Since the semiclassical evolution of this reflecting solution is unitary by construction [13, 14, 5, 3], the radiation to the future of $u_{0}$ must be strongly correlated with the radiation to the past of $u_{0}$. To see this explicitly we calculate the correlation function

$$
C_{\mu \nu, \mu^{\prime} \nu^{\prime}}\left(x, x^{\prime}\right) \equiv\left\langle T_{\mu \nu}(x) T_{\mu^{\prime} \nu^{\prime}}\left(x^{\prime}\right)\right\rangle-\left\langle T_{\mu \nu}(x)\right\rangle\left\langle T_{\mu^{\prime} \nu^{\prime}}\left(x^{\prime}\right)\right\rangle
$$

We are especially interested in $C_{--,--}\left(u, u^{\prime}\right)$ which describes the correlations in the outgoing radiation on $\Im^{+}$. For reflecting boundary conditions there is a closed form expression [4] : 


$$
C_{--,--}\left(u, u^{\prime}\right)=\frac{1}{8 \pi^{2}} \frac{\left(\partial_{u} v_{B}(u)\right)^{2}\left(\partial_{u^{\prime}} v_{B}\left(u^{\prime}\right)\right)^{2}}{\left(v_{B}(u)-v_{B}\left(u^{\prime}\right)\right)^{4}}
$$

where $v_{B}(u)$ is the boundary curve in the coordinates $v=\lambda^{-1} \log \left(\lambda x^{+}\right)$and $u=$ $-\lambda^{-1} \log \left(-\left(\lambda x^{-}+P_{+} / \lambda\right)\right)$. Let us first consider the correlations in the radiation reaching $\Im^{+}$before the classical reflected matter. For $u<u_{0}$ we have $v_{B}(u)=\lambda^{-1} \log \left(-\lambda^{2} x_{0}^{+} x_{0}^{-}\right)-$ $\lambda^{-1} \log \left(e^{-\lambda u}+P_{+} / \lambda\right)$, and for $M$ near $M_{c r}$ we have $e^{\lambda u_{0}}>>1$. Taking $u<u_{0}$ and $u^{\prime}<u_{0}$, with both near $u_{0}$, we find to leading order in $\lambda e^{-\lambda u} / P_{+}$and in $\lambda e^{-\lambda u^{\prime}} / P_{+}$,

$$
C_{--,--}\left(u, u^{\prime}\right)=\frac{\lambda^{4}}{8 \pi^{2}} \frac{\left(e^{-\lambda u}\right)^{2}\left(e^{-\lambda u^{\prime}}\right)^{2}}{\left(e^{-\lambda u}-e^{-\lambda u^{\prime}}\right)^{4}}\left(1+\mathrm{O}\left(\frac{\lambda e^{-\lambda u}}{P_{+}}\right)\right) .
$$

The leading term in (10) is just the correlation function in 2D for thermal black body radiation 四 with temperature $T=\lambda / 2 \pi$, the temperature of $2 \mathrm{D}$ dilatonic black holes [15,16]. Through the same calculation that gave Eq. (10), we find that (10) is also the correlation function for Hawking radiation from an evaporating 2D black hole.

To explicitly verify the correlations between the quantum radiation reaching $\Im^{+}$before and after the classical reflected matter, we numerically calculate (9) by holding $u^{\prime}$ fixed on either side of $u_{0}$ and plotting $C_{--,--}\left(u, u^{\prime}\right)$ as a function of $u$. The results are shown in Fig. 因, where the mass of the infalling matter is just below the critical mass, $M=0.99 M_{c r}$.

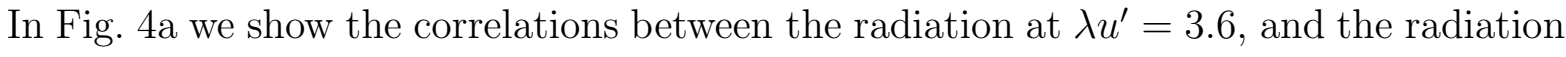
elsewhere on $\Im^{+}$. This value of $\lambda u^{\prime}$ is sufficiently close to $\lambda u_{0}(=4.6)$ that the radiation has the same form as the Hawking radiation from a black hole. For $u<u_{0}$ the correlation is almost thermal, as in Eq. (10). It diverges at $u=u^{\prime}$ and becomes small for $\left|u-u^{\prime}\right| \sim 1 / \lambda$. The most dramatic effect occurs just after the reflected matter reaches $\Im^{+}$, i.e., at $u=u_{0}+\delta$. The correlations rise (continuously when $\epsilon$ is finite [10]) to extremely high values of order $e^{15}$. These huge correlations are required for unitary evolution because the negative-energy radiation reaching $\Im^{+}$after the classical reflected matter is very localized in time.

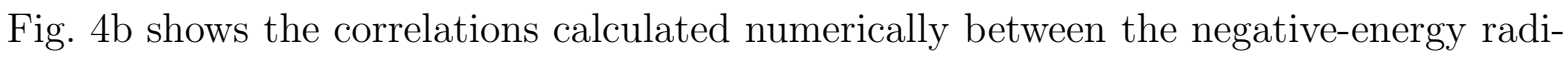
ation reaching $\Im^{+}$just after the classical reflected matter and the radiation elsewhere on $\Im^{+}$. In this figure we take $\lambda u^{\prime}=\lambda u_{0}+\delta$ and $\delta \rightarrow 0^{+}$. The correlations diverge at $u=u^{\prime}$ 
and decrease rapidly when $u>u^{\prime}$. On the other hand the correlations with the Hawking radiation $\left(u<u_{0}\right)$ remain extremely high for relatively large values of $\lambda\left|u-u^{\prime}\right|$. In contrast, if one were to take $\lambda u^{\prime}>>\lambda u_{0}$ then the only significant correlations would occur for $\lambda u$ near $\lambda u^{\prime}$.

The above results may plausibly be interpreted as arising from the creation of particleantiparticle pairs [17]. The particles reach infinity and give rise to the positive (Hawking) radiation, while the antiparticles carrying negative energy are reflected from the boundary and give rise to the negative energy radiated to $\Im^{+}$after the classical reflected matter. If it were not for the negative-energy burst the correlations between the particles and antiparticles would be lost and the final state would be a mixed state. Thus, the negative-energy burst can be regarded as carrying information equal to the magnitude of the entropy $\mathcal{S}$ of the Hawking radiation reaching $\Im^{+}$ahead of the classical reflected matter. As $M$ approaches $M_{c r}$, where $M_{c r}>>\kappa \lambda / 4$, we find that $\mathcal{S} \approx(N / 12) \log \left[4 M_{c r} /(\kappa \lambda)\right]$. The negative-energy burst thus carries information to $\Im^{+}$at the rate $\dot{I}=\mathcal{S} / \Delta t$. Using the earlier upper bound on $|E| \Delta t$, we find that this gives $\dot{I} \geq(N \lambda / 48)\left(1-M / M_{c r}\right)^{-2}$. The theoretical upper bound on the bulk rate of linear information flow in $N$ channels for $E>0$ is $[18] \dot{I}_{\max } \leq[E /(2 \pi \hbar)] \log _{2}(N)$ to within a factor of order 1 . If this bound can be extended to $E<0$ by replacing $E$ by $|E|$, then it gives for the present system: $\dot{I}_{\max } \leq[N \lambda /(96 \pi)] \log \left[4 M_{c r} /(\kappa \lambda)\right] \log _{2}(N)$. This bound is clearly exceeded for large but finite $N$ in our system as $M$ approaches $M_{c r}$.

We thank Bruce Allen, John Friedman, Jorma Louko and Eli Lubkin for helpful discussions and especially Scott Koranda for help with the numerical analysis. This work was supported by the National Science Foundation under grant PHY 95-07740. 


\section{REFERENCES}

[1] S. Bose, L. Parker and Y. Peleg, "Semi-infinite Throat as the End-state Geometry of two-dimensional Black Hole Evaporation", UWM report no. WISC-MILW-95-TH-10, hep-th/9502098, to appear in Phys.Rev.D.

[2] S.A. Fulling and P.C.W. Davies, Proc.R.Soc.London, A348,393 (1976); N.D. Birrell and P.C.W. Davies, "Quantum Fields in Curved Space", (Cambridge Univ. Press, Cambridge, 1982).

[3] A. Strominger and L. Thorlacius, Phys.Rev.D50, 5177 (1994).

[4] R.B. Carlitz and R.S. Willey, Phys.Rev.D36, 2327, 2336 (1987); F. Wilczek, "Quantum Purity at a Small Price: Easing a Black Hole Paradox", Princeton-IAS report no. IASSNS-HEP-93-12, hep-th/9302096 (1993).

[5] T. Chung and H. Verlinde, Nucl.Phys.B418, 305 (1994).

[6] P.C.W.Davies, S.A. Fulling and W.G. Unruh, Phys.Rev.D13, 2720 (1976).

[7] J.L. Cardy, Nucl.Phys.B240, 514 (1984).

[8] J. Russo, L. Susskind and L. Thorlacius, Phys.Rev.D46, 3444 (1992).

[9] In 4D dilaton gravity, $X=e^{-2 \phi}$ corresponds to the radial coordinate, see Y. Peleg, Mod.Phys.Lett.A9, 3137 (1994). $X_{B}=0$ corresponds to the origin of coordinates.

[10] S. Bose, L. Parker and Y. Peleg, "Correlations and radiation for nearly collapsing matter in 2D dilaton Gravity", in preparation. In this work we study general infalling matter and show how the "limiting" case, $\lambda \epsilon \rightarrow 0$, is approached.

[11] William H. Press et. al. Numerical Recipes in C, 2nd ed. (Cambridge Univ. Press, Cambridge, 1992).

[12] L.H. Ford and T. Roman, "Averaged Energy Conditions and Evaporating Black Holes", MIT-Tufts report no. MIT-CTP-2446, gr-qc/9506052 (1995). 
[13] C.R. Stephens, G. 't Hooft and B.F. Whiting, Class.Quant.Grav.11, 621 (1994).

[14] A. Miković, Phys. Lett. B291, 19 (1992); B304, 70 (1993).

[15] E. Witten, Phys.Rev.D44, 314 (1991).

[16] C. Callan, S. Giddings, J. Harvey and A. Strominger, Phys.Rev.D45, R1005 (1992).

[17] L. Parker, Phys.Rev.D12, 1519 (1975); R.M. Wald, Comm.Math.Phys.45, 9 (1975).

[18] L.B. Levitin, Int. J. Theor. Phys. 21 (1982); J.D. Bekenstein and M. Schiffer, Int. J. Mod. Phys. C1, 355 (1990); H.J. Bremermann, Int. J. Theor. Phys. 21, 203 (1982). 


\section{FIGURES}

FIG. 1. Penrose diagram for a typical subcritical solution. The $2 \mathrm{D}$ space-time is the interior of the 1D null hypersurfaces $\Im^{-}, \Im^{+}$and the time-like boundary curve, which intersect at $i^{-}=\left(x^{+}=0, x^{-}=-\infty\right), i^{0}=\left(x^{+}=\infty, x^{-}=-\infty\right)$ and $i^{+}=\left(x^{+}=\infty, x^{-}=0\right)$.

FIG. 2. The boundary curve $\lambda x_{B}^{+}=q(y)$. In (a) the mass of the infalling matter is $M=0.1 M_{c r}$ and in (b) it is $M=0.9 M_{c r}$. The dimensionless coordinate $y=\lambda x^{-}+P_{+} / \lambda$ is plotted on the horizontal axes.

FIG. 3. Quantum radiation on $\Im^{+}$. In (a) $M=0.1 M_{c r}$ and in (b) $M=0.9 M_{c r}$. Here $\left\langle T_{--}\right\rangle$is in units of $\kappa \lambda^{2} / 4$ (which is the value for Hawking radiation from $2 \mathrm{D}$ black holes). In (a) $\lambda u_{0}=0.1$ and in (b) $\lambda u_{0}=2.3$.

FIG. 4. Log of the correlation function $C_{--,--}\left(u, u^{\prime}\right)$ for fixed $u^{\prime}$. In (a) we take $\lambda u^{\prime}=3.6$, and in (b) $\lambda u_{0}$ approaches $\lambda u_{0}=4.6$ from above. 
FIGURE 1

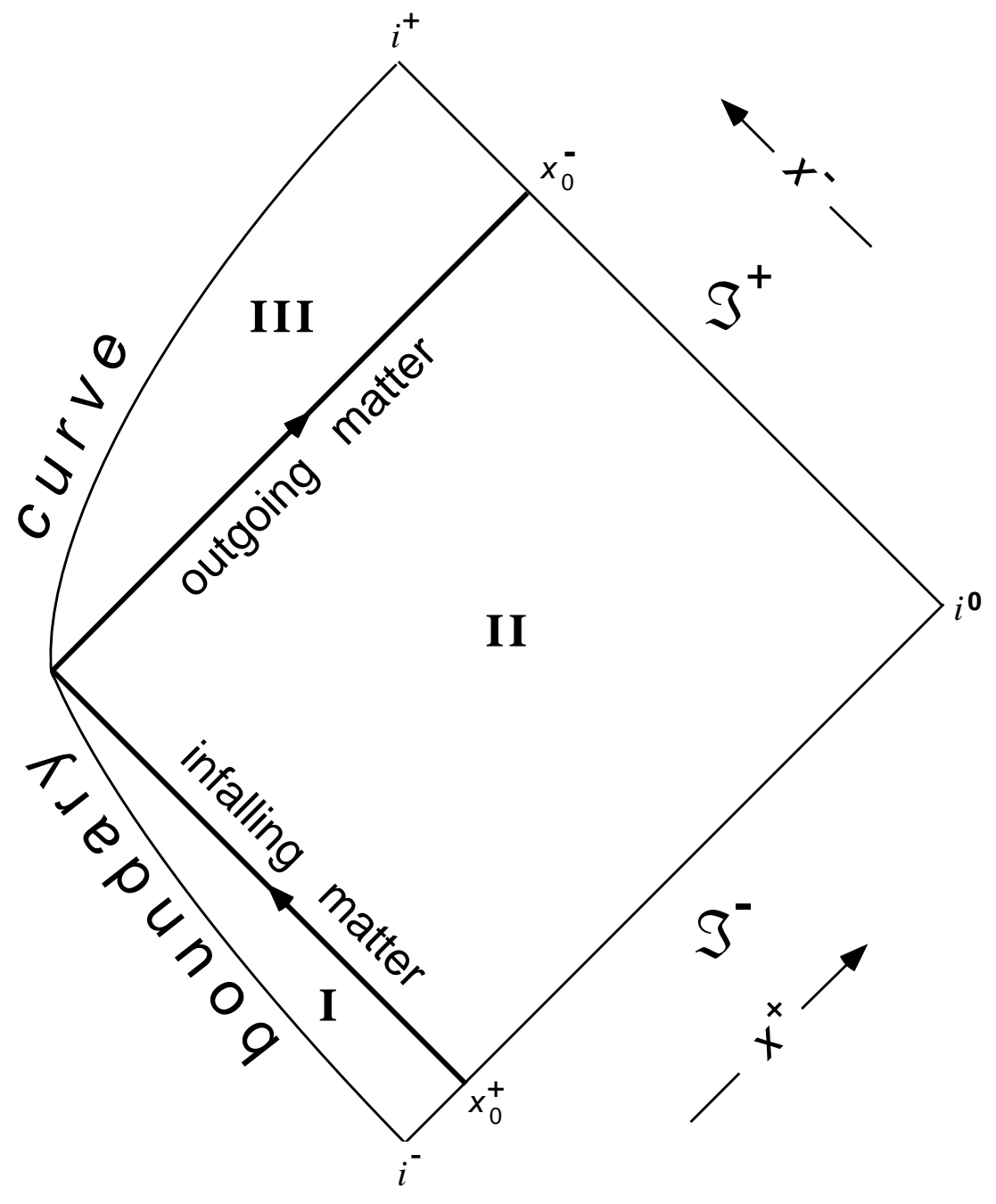


FIGURE 2
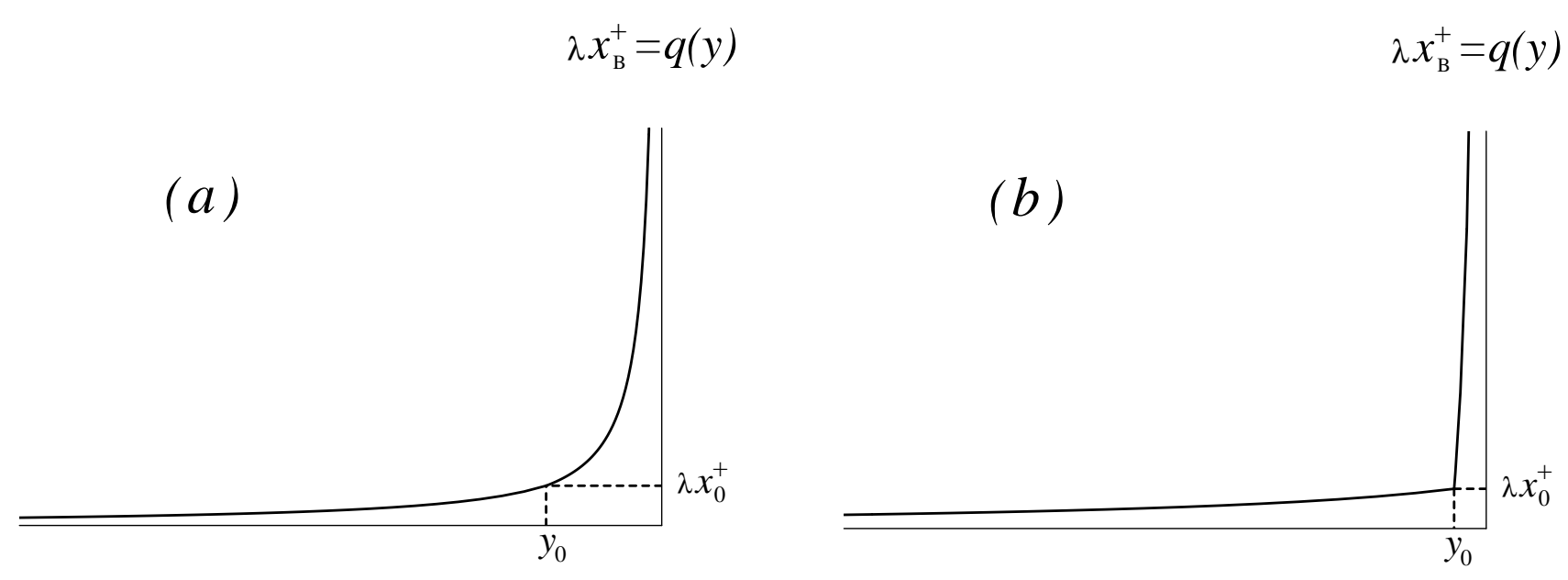
FIGURE 3
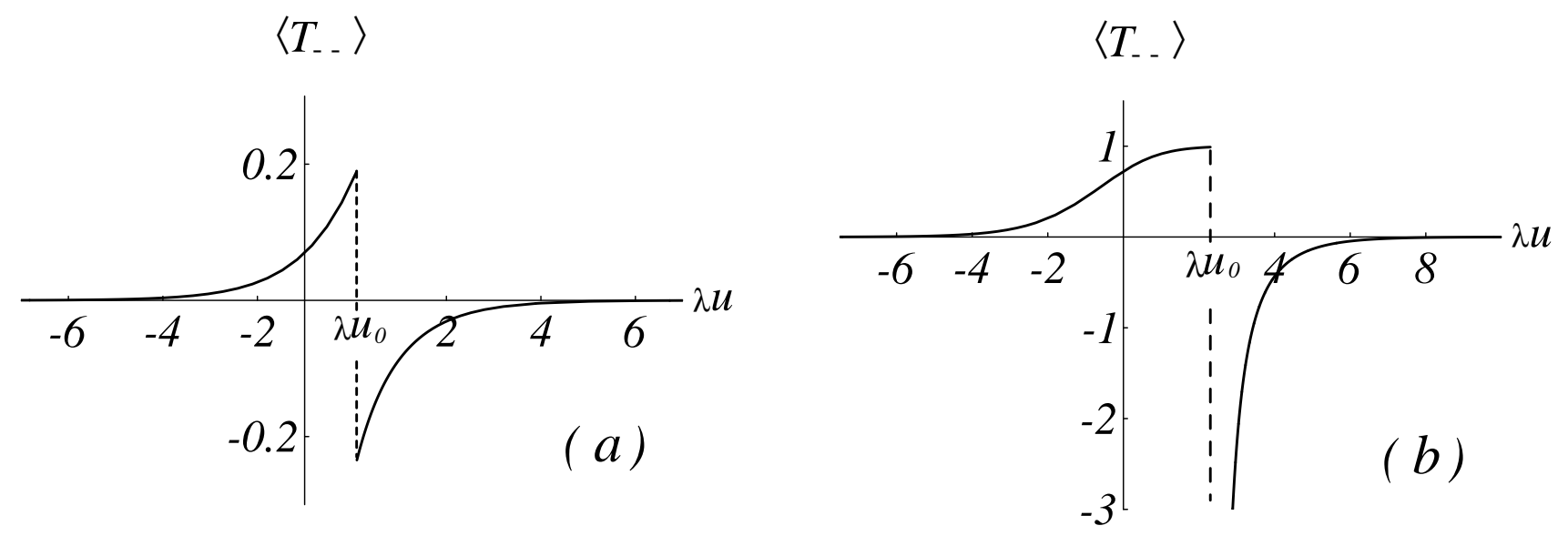
FIGURE 4

$\log \left(C_{-},-,-/ \lambda^{4}\right)$

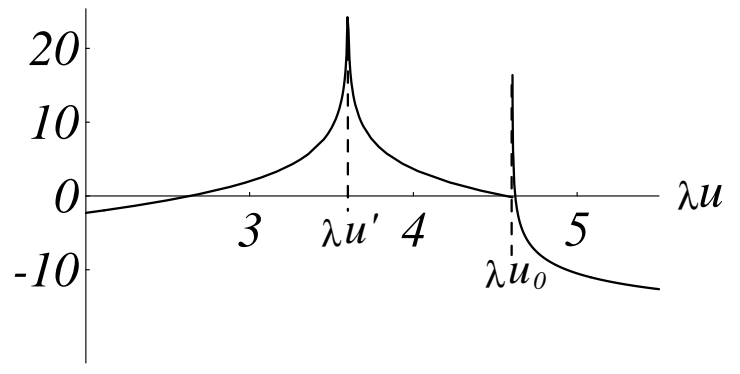

(a) $\log \left(C_{--,-}-/ \lambda^{4}\right)$

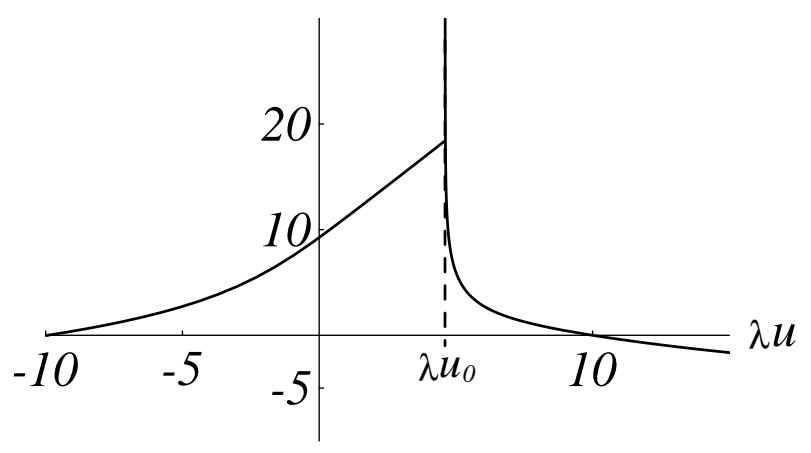

(b) 